\title{
Symbolische Diebstähle.
}

\author{
Von \\ Dr. A. Kielholz (Königsfelden).
}

Vortrag, gehalten an der 57. Sitzung des Schweiz. Vereins für Psychiatrie in Freiburg am 1. XI. 1919.

(Eingegangen am 12. Januar 1920.)

Wohl den meisten von uns ist es schon begegnet, daß sie einen Brief so verlegten, daß er nicht zur nützlichen Frist oder nur unter Aufwendung zeitraubender Mühe, vielleicht nach langem Suchen und Besinnen wieder gefunden werden konnte. Forschten sie dem Grunde dieses Verlegens nach, so ergab sich, daß die Persönlichkeit des Briefschreibers oder der Inhalt des Schreibens irgendwie unangenehme Gefühle erweckten, und in dem Verlegen des Briefes manifestierte sich die unbewußte Absicht, ihn nicht zu beantworten oder seinen Verfasser durch eine verzögerte Antwort irgendwie in Verlegenheit zu setzen oder zu strafen. Das Verlegen des Biefes erwies sich also mit anderen Worten als der symbolische Ausdruck einer Absicht oder eines Wunsches. Freud hat bekanntlich in seiner Psychopathologie des Alltagslebens sich um die Erklärung solcher Fehlhandlungen bemüht und ihre Deutung aus Mechanismen des Unbewußten versucht.

Man hat nun hier und da Gelegenheit, kriminelle Handlungen, insbesondere Diebstähle zu beobachten und zu beurteilen, die sowohl in Hinsicht auf die Persönlichkeit des Täters als auch auf die näheren Umstände der Tat nicht in das landläufige Schema solcher Delikte passen. Die zureichende Motivierung des Vergehens aus Charakter, Milieu und Gelegenheit fehlt entweder ganz oder ist nur recht mangelhaft $\mathrm{zu}$ konstruieren, der Täter selbst versichert bestimmt und in glaubwürdiger Weise, das Motiv selbst nicht zu kennen oder gibt Gü̈nde für seine Handlung an, die dem Beobachter recht unstichhaltig erscheinen. Erklärlich werden diese Diebstähle aber dadurch, daß man ähnliche unbewußte Mechanismen berücksichtigt, wie wir sie eben bei den sog. Fehlhandlungen berührt haben, wenn man also diese'Delikte ebenfalls als symbolische Betätigungen auffaßt. 
Es sei gestattet, an Hand einiger rasch skizzierter Fälle diese Auffassung darzulegen:

Die 36 jährige, alleinstehende, mit einem sprachfehler behaftete und schwerhörige Jungfer Katharine H. stahl einem Gemeinderat ihres Heimatsdorfes während der Nacht einen zweijährigen Stier aus dem Stall und fütterte und tränkte ihn im geheimen in ihrem Heimwesen. Zwei Wochen lang war man über die Täterschaft im unklaren und entdeckte das gestohlene Tier erst, als es die Diebin bei einem Metzger des Nachbardorfes, zu dem sie des Morgens noch im Finstern auf Umwegen gewandert war, verkaufen wollte. Bei einer Hausdurchsuchung, die nach Verhaftung der Jungfer H. vorgenommen wurde, fanden sich zwei Paar alte Militärhosen und ein Sack Zucker vor, die sie einem anderen Gemeinderat gestohlen hatte.

Bei der Begutachtung der Inkulpatin wurde festgestellt, daß ihre Großmutter mütterlichseits als Diebin bekannt war, sie selbst aber als eine brave, wenn auch etwas beschränkte Person galt, der niemand etwas Schlechtes zutraute. Sie habe sich lebhaft einen Mann gewünscht, aber keinon kriegen können, berichtete der Gemeindeamtmann und er verinutete auch, daß der Wunsch, ihre bescheidene ökonomische Lage zu verbessern, um dann eher heiraten zu können, sie zu diesen Delikten getrieben habe.

Diese Erklärung streift wohl die Wahrheit, zeigt uns aber die Unzulänglichkeit einer Motivierung, die nur mit bewußten Gründen rechnet. Was nützten der Jungfer Katharine die alten Soldatenhosen und der Zucker zur Aufbesserung ihrer Finanzen? Wir halten das Delikt für ein Musterbeispiel eines symbolischen Diebstahls, einer Wunschverwirklichung, bei welcher der junge Sties und die männlichen Kleidungsstücke wohl auch von solchen Psychologen als durchsichtige Symbole anerkannt werden, die nicht mit allen Finessen analytischer Deutungskunst durch Dick und Dünn gehen wollen. Als Beleg dafür, daß auch der Sack Zncker solcher Symbolik recht nahe steht, darf wohl an jene zahlreichen Volkslieder erinnert werden, wo von zuckersüßen Küssen. Münrlern und Schätzen gesungen wird.

Der Gutachter stellte fest, daß man es bei Jungfer $H$. weder mit einer Geisteskranken noch mit einer hochgradig Schwachsinnigen zu tum habe und daß diese wohl imstande sei, die Tragweite strafbarer Handlungen bis zu einem gewissen Grade zu bemessen. Er taxierte aber die Entwendung cines Stückes Großvieh durch eine alleinstehende Frauensperson als recht imbezille Handlung, ebenso ihre Versuche es wieder loszuwerden und der Hosendiebstahl ersehien ihm vollends zwecklos und einfältig. Das Gericht verurteilte die Delinquentin zu 10 Monaten Zuchthaus. Etwa 12 Jahre später erkrankte sie an cinem Paranoid, das zur dauernden Anstaltsversorgung führte. Sie fühlte sich besonders nachts durch Telephonstimmen verfolgt, die in erster Linie von einem der Gemeinderäte herrührten, den sie seinerzeit bestohlen hatte und beklagte sich, der mache nachts mit ihr, was er wolle!

Die im Klimakterium aufgetretene Psychose erfüllte ihr also halluzinatorisch den Wunsch, dem sie durch ihren Diebstahl zur Zeit der sexuellen Reife symbolisch Ausdruck gegeben hatte.

Bei einem zweiten Falle ist es nicht direkt erotische Begehrlichkeit, die sich auf solche Weise auszuwirken sucht, sondern eher sexueller Neid oder Eifersucht.

Ein Leutnant von 21 Jahren entwendete seinem Zimmerkameraden, mit dem er schon manchen Militärdienst gemeinsam absolviert, in der ersten Nacht einer eben begonnenen Rekrutenschule einen Geldbeutel, der 25 Fr. und zwei Koffer- 
schlüssel enthielt. Am anderen Morgen half er ihm beim Untersuchen des Zimmers, gestand dann das Vergehen und gab ihm das Geld zurück. Den Geldbeutel mit den beiden Schlüsseln hatte er zum Fenster hinausgeworfen.

Vor Untersuchungsrichter und später während der psychiatrischen Beobachtung beteuerte der junge Mann immer wieder, er wisse selbst nicht, warum (rr das getan habe und sei nicht imstande, das Motiv seiner Handlung anzugeben.' Fr war auch nachweisbar nicht in Geldverlegenheit, hatte keine Schulden, konnte von daheim jederzeit Geld erhalten, war weder in Zivil noch beim Militär vorbestraft und wurde von seinen Vorgesetzten als vielversprechender Soldat qualifiziert, der trotz seiner Jugend in rascher Folge die verschiedenen Kurse zur Erlangung seines Grades absolviert hatte. In der Aspirantenschule war er dann in allzu trinklustige Gesellschaft geraten, hatte aber vor dem eben angetretenen Dienst ein halbes Jahr im Zivilleben sich in dieser Beziehung solid verhalten. Am Abrnd vor dem Delikt beteiligte er sich an einer Bierreise mit seinen Kameraden. In einer Animierkneipe bemühten er und der Leutnant, den er nachher bestahl, sich um die Gunst einer Kellnerin. Sein Partner hatte dabei mehr Glück und unser Delinquent zog sich verstimmt in sein Zimmer zurück und legte sich schlafen, wurde aber nach $11 / 2$ Stunden von den heimkehrenden, angetrunkenen Kameraden geweckt und geneckt. Er wartete, bis diese ruhig geworden und eingeschlafen waren und entwendete dann aus den über einen Stuhl gehängten Beinkleidern seines Zimmergenossen den Geldbeutel.

Der Dieb versicherte uns Ärzten, noch nie sexuellen Verkehr gepflogen zu haben. Er sei zwar vor einem Jahre verlobt gewesen, habe aber das Verhältnis nach kurzer Zeit wieder gelöst, habe auch viel geflirtet und wie andere mit seinen Wrfolgen beim weiblichen Geschlecht geprahlt, ohne aber tatsächlich je solche errungen zu haben.

Unter solchen Umst:̈nden scheint die Deutung erlaubt, der Leutnant habe seinen Kameraden, der bei den Weibern so rasch zum Ziel gelangte, um seine sexuelle Tüchtigkeit beneidet und ihn in dem Geldbeutel mit den zwei Kofferschlüsseln darin symbolisch seiner Potenz berauben wollen. Ein 'Traum, den er dem Referenten erzählte, bestätigte diese Deutung: Er sei dem bewußten Kameraden begegnet und habe mit dem eigenen Säbel des anderen Schlagband abgeschnitten. Das umschreibt in kaum verhültem Bild ebenfalls eine Kastrationshandlung.

Das Gutachten erklärte den erblich schwer belasteten Exploranden als Psychopathen und Neurastheniker - er hatte auch in der Untersuchungshaft einen ernsthaften Suicidversuch unternommen - für seine triebartige Handlung als unzurechnungsfähig, doch verurteilte ihn das Militärgericht trotzdem zu 3 Monaten Gefängnis, einem Jahr Einstellung im Aktivbürgerrecht und Versetzung zu den Ersatzpflichtigen.

In einem dritten Falle ist es im Gegensatz zu den beiden ersten nicht zu einer gerichtlichen Beurteilung gekommen, und es haben sich daher weder Ärzte noch Richter ïber die Frage der Zurechnungsfähigkeit aussprechen müssen.

Es handelte sich um die 37 jährige Gattin eines Zahnarztes, die wegen Hysterie mit heftigen Angstanfällen und ernsthaften Suicidversuchen interniert werden mußte. Sie hatte sich mit einem heimlich entwendeten Tischmesser am Handgelenk eine tiefe Schnittwunde beigebracht, daß eine starke Blutung erfolgte und die Unterbindung der Radialarterie notwendig wurde. Auch jetzt versuchte sie beständig Seheren und Tischmesser zu entwenden, oft mit Erfolg, versteckte dann die entwendeten Instrumente im Bett oder im Sofa und konnte sich einmal mit 
einem Messer am Handgelenk kritzen. Zur Rede gestellt, erklärte sie, es ganz motivlos getan zu haben, aus Freude oder Spannung, ob man es wohl entdecken werde, dann wieder sagte sie, der Diebstahl eines Dessertmessers habe sie in ihrer Angst beruhigt, ein anderes Messer würde ihr keine Beruhigung gewähren. Diese Angst spürte sie jeweils zuerst im Bauch, dann zum Herz aufsteigen, dann klagte sie über Stirnkopfschmerzen. Sie schuldigte sich als eine verworfene Person selber an, weil sie geschlechtlich gereizt sei, masturbiere, und befürchtete, rückenmarksschwindsiichtig und unheilbar geisteskrank zu sein. Dabei erwies sich Gedächtnis und Intelligenz als intakt. Die Anamnese und Analyse ergab, daß die Frau seit Jahren unter der sexuellen Anspruchslosigkeit und zeitweisen Impotenz ihres viel älteren Gatten gelitten hatte. Es sei ihr am Morgen oft zum Verzweifeln gewesen, wenn sie immer wieder vergeblich gehofft, es komme zum Coitus. Ein Jahr nun vor ihrer Internierung war sie eines Abends an einer abgelegenen Straßenecke einem Exhibitionisten begegnet, habe, weil sie kurzsichtig war, aus der Nähe hingestarrt und gesehen, wie der Mann eine Erektion bekam. Sie sei dann voll Schrecken fortgerannt und habe sich heftig aufgeregt. Kurz nachher, während einer Erholungskur, saß sie einem Herrn gegenüber, welcher, wenn jemand über ein körperliches Übel klagte, ein Scherchen und sein Dessertmesser vor sich hinlegte und den stereotypen Witz machte: Da haben sie eine Schere und ein Messer zur Auswahl, schneiden sie das Glied ab, das Sie ärgert. Sie habe dabei oft gedacht, oh, wenn ich den Gedanken, der mich quält, nur aus dem Gehirn herausschneiden könnte. Nachdem der Pat. der Zusammenhang ihrer Angstanfälle sowie der Messer- und Scherendiebstähle mit den früheren Erlebnissen und dem Verhältnis zum Mann durch die Analyse klargemacht worden, trat eine rasche Besserung ein, sie konnte lebenslustig und arbeitsfähig entlassen werden und blieb es 6 'Jahre lang. Dann gab der Umstand, daß sie ihren Gatten bei einem zärtlichen Tête-à-tête mit einem Empfangsfräulein ertappte, den Anstoß zur Wiedererkrankung mit ganz ähnlichen Symptomen. Es erwies sich nun, daß sie ihren Mann, der sehr sparsam war, schon seit langem mit Rechnungen bemogelt, sich einen zweiten Schlüssel zu seinem Kassenschrank verschafft und ihn, immer unter heftiger Angst, daraus bestohlen hatte. Das Geld verwendete sie für Sofakissen, Wandbilder und kunstgewerbliche Gegenstände, um sich ein schönes Heim zu schaffen. Sie beging diese Delikte, seit sie vom jüngeren Bruder ihres Mannes verfolgt und einmal geküßt worden war. Dieser Schwager hatte eine kunstsinnige Gattin, welche der Pat. ganz im Anfang der Ehe einmal erklärte, sie sei aus reiner Liebe geheiratet worden, von der Pat. könne man das nicht sagen. Mit der Verwendung des gestohlenen Geldes suchte diese also ihre Schwägerin, die sie innerlich nicht mochte, zu übertrumpfen, sie wollte es ebensogut oder noch besser haben und gönnte ihr den Mann nicht. Gleichzeitig träumte ihr häufig unter starker Angst, ihr eigener Mann sei verunglückt, werde ihr tot ins Haus gebracht, sie wolle sich von ihm scheiden lassen.

Der unbewußte Wunsch nach Beseitigung des Mannes, der in diesen Träumen zum Ausdruck kam, paßt garz zu der Tendenz der Diebstähle, sich an die Stelle der kunstsinnigen Schwägerin, die aus Liebe geheiratet wurde, zu setzen. Das Delikt erweist sich also auch hier vor allem als symbolische Handlung.

Nach mehrmonatigem Aufenthalt in der Anstalt gelang es der Kranken, die Wachsamkeit ihrer Pflegerin zu täuschen, zu entweichen und offenbar in einem Angstanfall Suicid zu begehen.

Die angeführte Kasuistik genügt wohl trotz ihrer Kürze, um einer zureichenden Begriff davon zu geben, jwas unter symbolischen Diebstählen verstanden werden soll. Es ist kein Zufall und beruht nicht auf 
einer Absicht oder einseitigen Betrachtungsweise des Referenten, daß in allen drei Fällen ein deutlicher Zusammenhang besteht zwischen Delikt und Sexualität des Täters. Dieser Zusammenhang ist schon längst bekannt. Ich verweise nur auf die Arbeit Oberholzers im Archiv für Kriminalanthropologie 50, über dieses Thema, wo sogar in der Kasuistik der Beweis für den innigen Konnex von Geschlechtstrieb und Eigentumsdelikten bei gewissen Individuen durch die günstige Wirkung der Kastration geleistet wird.

Thre Genese aus sexuellen Triebkräften weist den symbolischen Diebstählen eine nahe Verwandtschaft mit gewissen Vergehen von sexuell Perversen zu, die sich ebenfalls in triebhafter Weise ohne Rücksicht auf die Gefahr, mit den Strafgesetzen in Konflikt zu kommen, Gegenstände verschaffen, welche in symbolhafter Weise zur Befriedigung ihrer Lust dienen. Ich erinnere an die Zopfabschneider, die Kleiderund Schuhfetischisten. Bei allen diesen Leuten 'stellt doch der Fetisch ursprünglich ein Symbol dar für eine geliebte Person, bildet eine Reminiszenz an eine erotische Szene, bei der sich aus gewissen konstellativen Momenten die Neigung an eben diesen Gegenstand allein fixierte.

Auf der anderen Seite berühren sich die symbolischen Diebstähle mit Symbolhandlungen nicht krimineller Natur, wie sie beispielweise bei Zwangsneurotikern häufig beobachtet werden, wo durch gewisse, dem Bewußtsein des Betreffenden unverständliche Zeremonien und Betätigungen ebenfalls eine unbewußte Tendenz symbolisch zur Darstellung kommt. Auf die Ähnlichkeit der Phänomene unseres Themas mit den sog. Fehlhandlungen des alltäglichen Lebens ist schon in der Einleitung hingewiesen worden.

Die hier versuchte Isolierung der Symboldiebstähle und der Hinweis auf ihre Verwandtschaft mit gewissen Handlungen von Neurotikern, Perversen und Normalen, möchte in erster Linie zur psychologischen Klärung von ähnlichen Vergehen, die auf den ersten Blick unbegreiflich erscheinen, anregen.

Ob eine psychanalytische Behandlung solcher Delinquenten die Neigung zu ihren Delikten unter Umständen günstig beeinflussen oder sogar zum Schwinden bringen könnte, wagen wir nicht zu behaupten, wenn auch die langjährige Besserung der Angsthysterie in unserem dritten Fall dafür spricht. Ein Vorgehen in dieser Richtung erscheint aber schon aus heuristischen Gründen angezeigt.

Der Boden, auf dem sich der symbolische Diebstahl entwickelt hat, ist in jedem der angeführten Fälle ein anderer: einmal ein mäßiger Schwachsinn, einmal eine Psychopathie und einmal eine Hysterie, in allen drei Fällen also kein Leiden, das primär schon Unzurechnungsfähigkeit bedingte, und wir sehen auch, daß beidemal, wo es zur gericht- 
lichen Verhandlung kam, der Richter nicht eine Aufhebung des freien Willens anerkennen wollte und den Diebstahl bestrafte.

Wenn wir nun berücksichtigen, daß wir alle die Handlungen ähnlicher Natur, die wir eben erwähnt haben, als rein triebhafte, aus dem Unbewußten motivierte, dem Bewußtsein des Täters gänzlich unverständliche, beurteilen müssen, so verdienen ohne Zweifel auch diese Symboldiebstähle eine analoge Wertung und erscheint daher die Auffassung gerechtfertigt, es sei ein Diebstahl, der sich psychologisch als eine symbolische Handlung erweist, als Delikt eines für diese Tat Unzurechnungsfähigen $\mathrm{zu}$ taxieren. 